\title{
A Study on Incidence of Clinical Hypoxic Ischemic Encephalopathy Injury in Newborns with Perinatal Asphyxia
}

\author{
Ritambhara Lohan, Gauri Chauhan*, Ashish Dalal, SP Gupta, AK Bhardwaj, Muskaan Ahlawat \\ Department of Dermatology \& Venerology, M.M Institute of Medical Sciences, Mullana, Ambala-133207
}

Received: July 15, 2016; Accepted: July 20, 2016; Published: July 25, 2016

*Corresponding author: Dr. Gauri Chauhan MD, Senior Resident, Department of Pediatrics, MMIMSR, Mullana, Ambala, India, Tel: 0+918-396-923736; Email:gaurichauhan31@gmail.com

\begin{abstract}
Background: Central nervous system injury associated with perinatal asphyxia is called as Hypoxic Ischemic Encephalopathy (HIE). HIE is the most important neurological problem occurring in the perinatal period.

Objective: To evaluate newborns with birth asphyxia based on APGAR scores and to correlate it with development of clinical HIE.

Method: A prospective clinical study of 50 asphyxiated newborns. The assessment of birth asphyxia was performed using 1 minute APGAR score $<5$ and/or 5 minutes APGAR socre $<7$. The neurological assessment of these 50 asphyxiated newborns was done using Sarnath and Sarnath Staging of HIE. The correlation between birth asphyxia and HIE was assessed.

Results: HIE occurred in 21/50 (42\%) asphyxiated newborns. According to HIE staging, HIE-I cases were 5 (23.81\%), HIE-II were $7(33.33 \%)$ and HIE-III were 9 (42.86\%). Death occurred in 7 (33.33\%) cases of HIE. There was no statistical significant correlation between HIE with gender, weight for gestation, maternal factors and foetal factors. However significant correlation between HIE-II with gestation $(\mathrm{p}<.05)$ and HIE-III with NVD and LSCS $(\mathrm{p}<.05)$ was noted. There was also significant correlation between Apgar score at 1 minute with HIE-III $(\mathrm{p}<.05)$ and Apgar score at 5 minutes with HIE-II $(\mathrm{p}<.02)$ and HIE-III $(\mathrm{p}<.05)$.

Conclusion: Most newborns with birth asphyxia did not develop HIE. But those who did develop had severe HIE-III. There was significant correlation between severity of birth asphyxia and development of severe form of HIE. As the 1 minute and 5 minutes Apgar score decreased the severity of HIE increased.
\end{abstract}

Keywords: Asphyxia; APGAR score; Encephalopathy; Perinatal; Weight

\section{Introduction}

Perinatal asphyxia refers to a condition during the first and second stage of labour in which impaired gas exchange leads to foetal hypoxemia, hypercarbia and metabolic acidosis [1, 2]. Perinatal asphyxia is a major factor contributing to perinatal and neonatal mortality [3]. Cerebral palsy is the most important long term outcome of birth asphyxia and may be accompanied with mental retardation, seizure disorder or other neurological and sensory (visual or auditory) disabilities [4]. It is the fifth largest cause of under-5 deaths (8.5\%) after pneumonia, diarrhoea, neonatal infections and complications of preterm birth [3]. In 1952, Dr. Virginia Apgar devised a scoring system that was a rapid method of assessing the clinical status of the newborn infant at $1^{\text {st }}$ minute of age [5]. It was designed to be a guide to the need for resuscitation of newborns and developed as an objective tool that measured five signs of physiologic adaptation. This scoring system quickly gained near universal acceptance. The Apgar score can somewhat predict mortality, however, it is not a tool to be used alone in determining neurologic outcomes of infants who survive [6].

Most of the births in developing countries occur at home usually attended by untrained birth attendants. Failure to initiate and sustain breathing immediately after delivery has been associated with severe foetal hypoxia or ischemia which can manifest in newborn as encephalopathy and may result in neonatal death or permanent motor and mental disability [3]. This injury has been termed as Hypoxic Ischemic Encephalopathy (HIE). The foetus who experiences a significant hypoxia episode is at risk of developing HIE or other end organ damage and its sequelae [7]. HIE is of concern in an asphyxiated neonate because it can lead to serious long term neuromotor sequelae among survivors [7]. The HIE score based on modified Sarnath scoring system is a clinical tool comprising of a set of clinical signs associated with CNS dysfunction and is used to assess status of a child following birth asphyxia [8]. Several new technologies like CT, MRI have become available to determine cerebral damage during perinatal period and predict long term neurological outcome. These modalities are however not readily available in many neonatal units in developing countries like India and hence the centres heavily rely on clinical assessment and scoring systems to predict HIE or neurological injury [9]. The present study tries to note the frequency of HIE in asphyxiated newborns based on the current neurological scoring system and tries to evaluate the prediction of degree of neurological injury based on severity of asphyxia sustained.

\section{Material and Methods}

The prospective clinical study was carried out in neonatal unit of Maharishi Markandeshwar Institute of Medical Sciences 
and Research, Department of Pediatrics in collaboration with Department of Obstetrics and Gynaecology. The study included 50 neonates meeting the criteria mentioned below:

\section{Inclusion criteria}

- All neonates with birth asphyxia born in Labour Unit of our hospital.

- Exclusion criteria:

- Outborns

- Inborn neonates with congenital malformations, infections, chromosomal abnormalities, inborn errors of metabolism, dysmorphic syndromes and still born.

Birth asphyxia was defined using Apgar score at 1 minute $<5$ and/or Apgar Score at 5 minutes $<7$. Complete obstetric history was obtained and examination of the babies was performed after delivery. The neonatal clinical course was followed up prospectively and data was recorded on pre-designed proforma. Informed written parental consent was obtained for all infants before entry in the study, which was approved by the ethical committee.

Detailed neurological examination of asphyxiated newborns was performed after 24 hours of birth until discharge or death. The stage of encephalopathy was assessed according to Sarnath and Sarnath Clinical staging of HIE. Depending on the neurobehavioral signs neonates were divided in 3 stages I, II and III (Mild, moderate and severe). Further, the relationship between birth asphyxia and HIE was also studied in all included newborns.

\section{Statistical Analysis}

Appropriate data entry and statistical analysis were performed on Microsoft excel and SPSS software. Data was summarized using descriptive statistics. Categorical variables are presented as number and percentage. Chi-square test was used to compare the association among two or more categorical variables. All statistical tests were two-tailed and alpha level of significance was set less than $5 \%$. A p value of $<0.05$ was taken as statistically significant.

\section{Results}

\section{Demographic and Clinical profile}

Among 50 newborns 30(60\%) were males and 20 (40\%) were females and $31(62 \%)$ were term and $19(38 \%)$ were preterm as per gestational age. As per weight for gestation, 42 (84\%) were appropriate for gestational age (AGA) and $8(16 \%)$ were small for gestational age (SGA). The various maternal, perinatal and foetal factors associated with asphyxia in newborns are highlighted in figure 1. 32/50 (64\%) were born by normal vaginal delivery (NVD) without instrumentation, 7 (14\%) with instrumentation and $11(22 \%)$ by lower segment caesarean section (LSCS). As per Sarnath and Sarnath clinical scoring for neurobehavioral signs and symptoms, 21 (42\%) had evidence of HIE. Upon grading, severe HIE (HIE-III) was most common, seen in 9/21 (42.86\%)

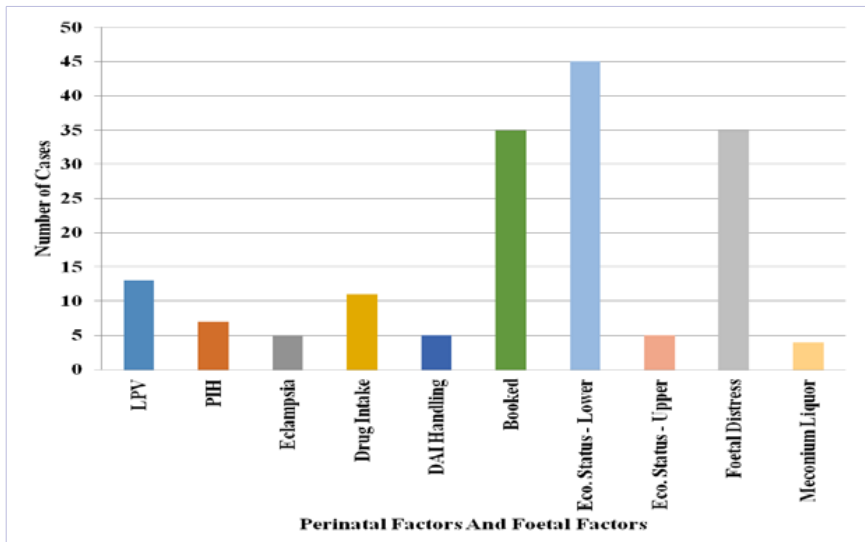

Figure 1: Histogram chart showing various fetal and perinatal factors related to asphyxia in newborns in present study.

cases table 1. Among 21 cases of HIE, death occurred in 7 $(33.33 \%)$ cases, $12(57.15 \%)$ were alive till last follow up and 2 $(9.52 \%)$ cases left against medical advice (LAMA).

\section{Correlation between HIE and APGAR score}

Tables 2 and 3 highlight correlation of HIE with APGAR scores at 1 and 5 minute respectively. There was a statistically significant correlation between HIE-III with APGAR at 1 minute $(\mathrm{p}=.05)$ noted as all 4 cases with an APGAR of 0 - 2 had severe HIE (HIE-III) as compared to none with a score of 5 and only $23.8 \%$ with a score of 3-5. There was also a statistically significant correlation noted between HIE-II $(p=.05)$ and HIE-III $(p=.01)$ with APGAR score at 5 minutes table 3.

There was no statistically significant correlation noted between HIE with gender, weight for gestation and maternal and foetal factors which may be due to more awareness, education, proper antenatal care and timely referral to higher centre. However, HIE-II did had a significant association with gestational age $(\mathrm{p}<0.05)$.

\section{Discussion}

This study was conducted to note the frequency of HIE in asphyxiated newborns based on the current neurological scoring system and to correlate the development of hypoxic ischemic encephalopathy in asphyxiated newborns various fetal, perinatal and maternal variables. Fifty asphyxiated newborns were studied, all of them fulfilling the inclusion criteria. Out of these asphyxiated newborns born in our hospital, males were $30(60 \%)$ and females were $20(40 \%)$ and as per gestation, 31 $(62 \%)$ were term and $19(38 \%)$ preterm. Dalal A et al [9] in their study had 168 (52.5\%) males and 152 (47.5\%) females and 87\% were term neonates and $13 \%$ preterm neonates. In a similar study by Dag et al [10] 20 (55\%) were males and 16 (44\%) were females and 32 (88.8\%) were term newborns and 4 (11.11\%) preterm newborns. In present study preterm newborns could be low due to less of preterm deliveries nowadays because of better antenatal care and visits and assessment. According to weight for gestation, AGA were $42(84 \%)$ and 8 (16\%) SGA in our study. This may be due to better antenatal care and follow up. $52.8 \%$ AGA 
Table 1: Distribution of HIE cases as per Sarnath and Sarnath Staging of HIE.

\begin{tabular}{|c|c|c|}
\hline HIE Classification & Number $\mathbf{( N = 2 1 )}$ & Percentage \\
\hline HIE I & 5 & 23.81 \\
\hline HIE II & 7 & 33.33 \\
\hline HIE III & 9 & 42.86 \\
\hline Total & 21 & 100 \\
\hline
\end{tabular}

Table 2: Correlation between HIE and APGAR score at 1minute.

\begin{tabular}{|c|c|c|c|c|}
$\begin{array}{c}\text { APGAR at 1 } \\
\text { Minute }\end{array}$ & $\mathbf{0 - 2}$ & $\mathbf{3 - 5}$ & $\mathbf{= 5}$ & Total \\
\hline HIE - I & $(0 \%)$ & $5(23.81 \%)$ & $(0 \%)$ & $5(23.81 \%)$ \\
\hline HIE - II & $(0 \%)$ & $5(23.81 \%)$ & $2(9.5 \%)$ & $7(33.33 \%)$ \\
\hline HIE - III & $4(19.05 \%)$ & $5(23.81 \%)$ & $(0 \%)$ & $9(42.86 \%)$ \\
\hline Total & $4(19.05 \%)$ & $15(71.43 \%)$ & $2(9.52 \%)$ & $21(100 \%)$ \\
\hline Inference & $\begin{array}{l}\text { HIE stage III is significantly associated with APGAR at } \\
1 \text { minute with p<0.05 tested with Chi Square Test and } \\
\text { p<0.01 for Pearson Correlation Test }\end{array}$ \\
\hline
\end{tabular}

Table 3: Correlation between HIE and APGAR score at 5 minutes.

\begin{tabular}{|c|c|c|c|c|}
\hline $\begin{array}{c}\text { APGAR at } \mathbf{5} \\
\text { Minute }\end{array}$ & $\mathbf{0 - 3}$ & $\mathbf{4 - 7}$ & $\mathbf{= 7}$ & Total \\
\hline HIE - I & $(0 \%)$ & $1(4.76 \%)$ & $4(19.05 \%)$ & $5(23.81 \%)$ \\
\hline HIE - II & $2(9.52 \%)$ & $0(0 \%)$ & $5(23.81 \%)$ & $7(33.33 \%)$ \\
\hline HIE - III & $3(14.29 \%)$ & $6(28.57 \%)$ & $(0 \%)$ & $9(42.86 \%)$ \\
\hline Total & $5(23.81 \%)$ & $7(33.33 \%)$ & $9(42.86 \%)$ & $21(95.24 \%)$ \\
\hline & $\begin{array}{c}\text { HIE stage II is significantly correlated with APGAR } \\
\text { at } 5 \text { minute with p<0.05 tested with Pearson } \\
\text { Inference }\end{array}$ & $\begin{array}{c}\text { Correlation Test. } \\
\text { HIE stage III is significantly associated with APGAR } \\
\text { at } 5 \text { minute with p<0.01 tested with Chi Square Test } \\
\text { and p<0.01 tested with Pearson Correlation Test. }\end{array}$ \\
\hline
\end{tabular}

and $47.2 \%$ SGA were found in study by Dalal et al [9].

In our study, frequency of Hypoxic Ischemic Encephalopathy was $42 \%$ (21/50) of cases of asphyxia. Dalal etal [9] study showed $32.5 \%$ cases of asphyxia having HIE. In a study by Futrakul et al [11] $26 \%$ cases were of HIE, while $28.6 \%$ cases of HIE were seen in a study by Thornberg et al [12]. Ondoa-Onama et al [13] noted $21.8 \%$ cases of HIE and Gonzalez de Dios et al [14] showed HIE frequency of $25 \%$ in asphyxiated newborns. This difference noted in various studies in the proportion of HIE following perinatal asphyxia were probably due to lack of generally accepted definition of asphyxia. It is possible that incidence of HIE also depends on sample size. It also indicates that the assessment of birth asphyxia using APGAR scoring system was not a good prognostic marker. Also APGAR score assessment could have been affected by maturity of preterm neonates. According to Sarnath and Sarnath Classification of HIE, out of 21 cases of HIE, 5 (23.81\%) were HIE-I, 7 (33.33\%) HIE-II and 9 (42.86\%) HIEIII. This assessment may be due to low values of 1 minute and 5 minute APGAR score which had caused the neonates to land up in stage II and stage III of HIE. So, with increasing severity of asphyxia, severity of HIE also increases meaning that if less is the APGAR score values more will be the chances of development of HIE. This was also evident from a strong correlation between HIE-III and low APGAR scores (0-2) at $1 \mathrm{~min}(\mathrm{p}<0.05)$. HIE-II and HIE-III were also significantly correlated with APGAR score at 5 minutes $(\mathrm{p}<.05 ; \mathrm{p}<.01)$, suggesting that lower the value of Apgar score at $5 \mathrm{~min}$, more are the chances of development of moderate and severe forms of HIE. Futrakul et al [11] and Ellis et al [2] also noted significant correlation between HIE and 1 and 5 minutes APGAR score $(\mathrm{p}<.009)$.

However, our study has its own limitations in terms of small sample size, hospital setting study, based on only clinical assessment of asphyxia and HIE and with limited follow up data.

\section{Take Home Message}

- APGAR score is a useful tool for assessing babies requiring immediate resuscitation and for predicting immediate outcome in busy units but its predictive value has been considerably weakened as a sensitivity marker for asphyxia.

- APGAR score at 5 minutes predicts the better outcome than 1 minute score. 10 minutes score should also be assessed to predict the better neurodevelopment outcome and to assess whether additional resuscitative efforts are required if APGAR score is 0 at 10 minutes.

- HIE scoring system should be used to assess outcome of asphyxiated newborns as sophisticated or expensive equipment is not required. But other parameters to assess HIE shouldalso beconsidered if required and also according to availability.

\section{References}

1. Ringer SA. Resuscitation in delivery room. In: Cloherty JP, Eichenwald EC, Hansen AR, Stark AR. Manual of Neonatal Care. $7^{\text {th }}$ ed. Philadelphia: Wolters Kluwer. 2012;53-55.

2. Ellis M, Costello A. Birth asphyxia, Apgar score and neonatal encephalopathy. Indian Pediatrics. 1997;34:975-979.

3. Padayachee N, Ballot DE. Outcomes of neonates with perinatal asphyxia at a tertiary academic hospital in Johannesburg, South Africa. South Africa Journal. 2013;7(3):89-94. DOI:10.7196/SAJCH.574.

4. Reddy R, Kumar Praveen. Follow up of neonates with perinatal asphyxia. J Neonatol. 2004;18:22-27.

5. Committee on Foetus and Newborn, American College of Obstetricians and Gynecologists and Committee on Obstetric Practice Pediatrics. American Academy of Pediatrics. 2006;117:1444.

6. Leuthner S, Das G. Low Apgar scores and the definition of birth asphyxia. Pediatr Clin N Am. 2004;51(3):737-745. DOI:10.1016/j. pcl.2004.01.016.

7. Aggarwal R, Deorari A, Paul VK. Post-resuscitation management of asphyxiated neonates, Neonatology Unit Department of Pediatrics, All India Institute of Medical Sciences. Indian J Pediatr. 2001;68(12):11491153.

8. Beken S, Aydin B, Dilli D, Erol S, Zenciroglu A, Okumus N. Can 
biochemical markers predict the severity of hypoxic ischaemic encephalopathy? The Turkish Journal of Pediatrics. 2014;56(1):62-68.

9. Dalal EA, Bodar NL. A study on birth asphyxia at tertiary health centre. Natl J Med Res. 2013;3(4):374-376.

10. Dağ Y, Fırat AK, Karakaş HM, Alkan A, Yakıncı C, Erdem G. Clinical outcomes of neonatal hypoxic ischemic encephalopathy evaluated with diffusion-weighted magnetic resonance imaging. Diagn Interv Radiol. 2006;12:109-114.

11. Futrakul S, Praisawanna P, Thaitumyanon P. Risk factor for Hyposicischemic encephalopathy in asphysiated newborn infant. J Med Assoc Thai. 2006;89:322-328.
12. Thornberg E, Thiringer K, Odeback A, Milson I. Birth Asphyxia; incidence, clinical course and outcome in a Swedish population. Acta Paediatr. 1995;84(8):927-932.

13. Ondoa-Onama C, Tumwine JK. Immediate outcome of babies with low Apgar score in Mulago Hospital, Uganda. East Afr Med J. 2003;80(1):22-29.

14. González de Dios J, Moya M. Perinatal asphyxia, hypoxic-ischemic encephalopathy and neurological sequelae in full-term newborns. Rev Neurol. 1996;24(132):969-976. 